\title{
Genetic control of neuronal activity enhances axonal growth only on permissive substrates
}

1 Francina Mesquida-Veny ${ }^{1,2,3,4}$, Sara Martinez-Torres ${ }^{1,2,3,4}$, Jose Antonio Del Rio ${ }^{1,2,3,4} \dagger$, Arnau

2 Hervera ${ }^{1,2,3,4} \dagger^{*}$

$3{ }^{1}$ Molecular and Cellular Neurobiotechnology, Institute for Bioengineering of Catalonia (IBEC), 4 Barcelona, Spain

$5 \quad{ }^{2}$ Department of Cell Biology, Physiology and Immunology, University of Barcelona, Barcelona, Spain

$6 \quad{ }^{3}$ Network Centre of Biomedical Research of Neurodegenerative Diseases (CIBERNED), Institute of

7 Health Carlos III, Ministry of Economy and Competitiveness, Madrid, Spain

$8{ }^{4}$ Institute of Neuroscience, University of Barcelona, Barcelona, Spain

9 †These authors share senior authorship

10 * Correspondence:

11 Corresponding Authors

12 ahervera@ibecbarcelona.eu

13 Keywords: Optogenetics, chemogenetics, regeneration, permissive substrate, neuronal activity.

14 Abstract

15 Neural tissue has limited regenerative ability, to cope with that, in the recent years a diverse set of

16 novel tools have been used to tailor neurostimulation therapies and promote functional regeneration

17 after axonal injuries. In this report, we explore cell-specific methods to modulate neuronal activity,

18 including optogenetics and chemogenetics to assess the effect of specific neuronal stimulation in the

19 promotion of axonal regeneration after injury. We found that opto- or chemogenetic modulations of

20 neuronal activity on both dorsal root ganglia and corticospinal motor neurons increase their axonal

21 growth capacity only on permissive substrates.

\section{Introduction}

23 Following injury, damaged axons from the central nervous system (CNS) degenerate and are unable to 24 regenerate, while surviving fibres, have a limited capacity to sprout and to re-establish lost connections, 25 leading to functional impairment (Fitch and Silver, 2008).

This failure of CNS axons to regenerate after injury is partly attributed to a hostile CNS environment for growth (Richardson et al., 1980; Huebner and Strittmatter, 2009; Cregg et al., 2014). The injury site is rich in growth inhibitory proteins including myelin-associated glycoproteins, and chondroitin sulfate proteoglycans (CSPG) while lacking trophic support for axon regeneration (Jones et al., 2003; Ohtake and Li, 2015; Sami et al., 2020). The limited intrinsic regenerative capacity of adult CNS axons also contributes to the failure of axon regeneration after injury (He and Jin, 2016; Curcio and Bradke, 2018; Mahar and Cavalli, 2018). Yet, in the last years there have been several studies approaching different aspects of the CNS physiology to both boost the intrinsic regrowth capacity and overcome the extrinsic inhibition (Wu et al., 2015; Anderson et al., 2018). 
35

36

37

38

39

40

41

42

43

44

45

46

47

48

49

50

51

52

53

54

55

56

57

58

59

60

61

62

63

During development, when CNS neurons still retain their intrinsic ability to regenerate after axotomy (Arlotta et al., 2005), neuronal activity critically determines their connectivity, particularly onto spinal targets (Friel et al., 2014). In this direction, neuronal activation has also been explored to try to increase the intrinsic capacity for axon regeneration as well as overcome the extrinsic inhibition. Practically, electrical stimulation has been shown to enhance regeneration of sensory axons after peripheral nerve or dorsal columns injury (Udina et al., 2008a; Goganau et al., 2018a) and sprouting of cortical axons into contralateral spinal cord gray matter after pyramidotomy (Carmel et al., 2010a, 2014; Carmel and Martin, 2014a).

In order to gain insight on the actual mechanisms underlying these improvements, specifically and remotely activating neurons through opto- and chemogenetics, has become the state-of-the-art approach for tailored activity modulation (Sternson and Roth, 2014; Deisseroth, 2015).

In this direction, the current study explores the therapeutic potential of modulating neuronal activity of sensory ascending (dorsal root ganglia (DRG)) and motor descending (corticospinal motor neurons (CSMN)) neurons, via opto- and chemogenetic stimulation in regulating axonal growth after injury.

We found that specific neuronal stimulation induced increased axonal growth capacity both in vitro and in vivo, and both in sensory and motor neurons, but only when in presence of permissive and trophic environments. Opto- or chemogenetic stimulation did not overcome the inhibitory signalling induced by myelin-associated glycoproteins and CSPGs.

\section{Materials and Methods}

\section{Mice}

B6.Cg-Tg(Thy1-COP4/EYFP)18Gfng/J (Thy1-ChR2) (Jackson Laboratories) (Arenkiel et al., 2007) or wild type (WT) C57BL/6J mice (Charles River Laboratories) ranging from 6-10 weeks of age were used for the experiments and were randomly divided into the different experimental groups. Mice were anaesthetized with isoflurane (5\% induction, $2 \%$ maintenance) during surgeries. For DRG neuronal cultures adult Thy1-ChR2 mice were used. Embryonic day 16.5 (E16.5) OF1 pregnant females were purchased from Charles River Laboratories, and the embryos were used for neuronal cortical cultures. All procedures were approved by the Ethics Committee on Animal Experimentation (CEEA) of the University of Barcelona (CEEA approval \#276/16 and 141/15).

\section{Dorsal root ganglia (DRG) neuronal culture}

DRGs from adult Thy1-ChR2 mice were dissected, washed in cooled Hank's balanced salt solution (HBSS; ThermoFisher Scientific), and enzymatically digested $(5 \mathrm{mg} / \mathrm{ml}$ Dispase II (Merck) and $2.5 \mathrm{mg} / \mathrm{ml}$ Collagenase Type II (ThermoFisher Scientific) in DMEM (ThermoFisher Scientific)) for $45 \mathrm{~min}$ at $37^{\circ} \mathrm{C}$. Next, the DRGs were resuspended in DMEM:F12 (ThermoFisher Scientific) media supplemented with $10 \%$ heat-inactivated Fetal Bovine Serum (FBS; ThermoFisher Scientific) and 1x B27 (ThermoFisher Scientific) and were mechanically dissociated by pipetting. After centrifugation, the resulting single cells were resuspended in culture media (DMEM:F12 media with 1x B27 and penicillin/streptomycin (P/S; ThermoFisher Scientific)) and plated in glass-coverslips (3000-4000 cells $/$ well) pre-coated with $0.1 \mathrm{mg} / \mathrm{ml}$ poly-D-lysine $\left(2 \mathrm{~h}, 37^{\circ} \mathrm{C}\right.$; Merck) and $2 \mu \mathrm{g} / \mathrm{ml}$ laminin (over-night $(\mathrm{O} / \mathrm{N})$; ThermoFisher Scientific) at RT (room temperature)). An additional incubation with $20 \mu \mathrm{g} / \mathrm{ml}$ 
CSPGs (Merck) was performed $\left(2 \mathrm{~h}\right.$ at $\left.37^{\circ} \mathrm{C}\right)$ in growth-inhibitory substrate experiments. Cells were allowed to grow for $24 \mathrm{~h}$ at $37^{\circ} \mathrm{C}$ in a $5 \% \mathrm{CO}_{2}$ atmosphere.

\section{Neuronal cortical culture}

E16.5 WT mice embryos were used for neuronal cortical cultures. Brains were extracted, washed in cooled 0.1M phosphate-buffered saline (PBS; ThermoFisher Scientific) containing $6.5 \mathrm{mg} / \mathrm{ml}$ glucose (Sigma Aldrich) (PBS-glucose) and the meninges excised. Both cortical lobes were dissected, sliced in a McIlwain tissue chopper (Capdem Instruments) and trypsinized for $15 \mathrm{~min}$ at $37^{\circ} \mathrm{C}$. The suspension was inactivated with normal horse serum (NHS; ThermoFisher Scientific), incubated with $0.025 \%$ DNAse (Roche) PBS-glucose for $10 \mathrm{~min}$ at $37^{\circ} \mathrm{C}$ and mechanically dissociated. Single cells were spun down and resuspended in Neurobasal ${ }^{\mathrm{TM}}$ (ThermoFisher Scientific) medium supplemented with $2 \mathrm{mM}$ glutamine (ThermoFisher Scientific), P/S, 6.5mg/ml glucose, $\mathrm{NaHCO}_{3}$ (Merck), 1x B27 and NHS 5\% and plated in poly-D-lysine $(0.1 \mathrm{mg} / \mathrm{ml})$ pre-coated microfluidic devices (see below). One day after seeding, culture media was changed, and NHS was excluded from the new media. Cells were maintained at $37^{\circ} \mathrm{C}$ in a $5 \% \mathrm{CO} 2$ atmosphere and culture media was changed every two days.

\section{Microfluidic devices}

The design used for microfluidic devices was a modification of a previously published device(Taylor et al., 2005; Sala-Jarque et al., 2020). The resulting device consists in 4 receptacles of $7 \mathrm{~mm}$ diameter, connected in pairs by a longitudinal compartment (cell body and axonal compartments) which are in turn interconnected by 100 microchannels $(10 \mu \mathrm{m}$ x $10 \mu \mathrm{m} \times 900 \mu \mathrm{m})$. The master was produced using standard photolithography techniques at IBEC Microfab Space. Poly(dimethylsiloxane) (PDMS; Dow) was used to prepare the devices by soft lithography, which were subsequently attached to glass bottom dishes using oxygen plasma treatment. Neurons were seeded in one of the larger compartments (cell body compartment) and allowed to extend their axons across the microchannels. Vacuum aspiration in the axonal compartment allowed complete axotomies (Taylor et al., 2005).

\section{In vitro lentiviral production and infection}

LV-EF1 $\alpha$-hChR2(H134R)-EYFP-WPRE (LV-ChR2) produced in our laboratory were used to introduce ChR2 expression in primary cortical neurons. For LV production, 293-FT (ATCC) cells were simultaneously co-transfected with three plasmids: pMD2.G (VSV-G envelope expressing plasmid), psPAX2 (lentiviral packaging plasmid), and pLenti-EF1 $\alpha$-hChR2(H134R)-EYFP-WPRE (transfer plasmid) in Opti-MEM (ThermoFisher Scientific) using Lipofectamine 2000 Transfection Reagent (). Transfection media was changed for culture media (Advanced DMEM (ThermoFisher Scientific) supplemented with $10 \% \mathrm{FBS}, 1 \% \mathrm{P} / \mathrm{S}$ and $0.5 \%$ glutamine) $6 \mathrm{~h}$ after. Media was recovered at $48 \mathrm{~h}$ and $72 \mathrm{~h}$ post-transfection, filtered and centrifuged at $1200 \mathrm{~g}$ to remove debris. The supernatant was then centrifuged at 26.000rpm for $2 \mathrm{~h}$ at $4^{\circ} \mathrm{C}$ in a Beckman conical tube containing a sucrose cushion (20\%) for purification, ant the viral pellet was finally resuspended in PBS-1\% BSA. Cortical neurons were infected at 4 days in vitro (DIV) for 24h, and high levels of YFP fluorescence could be observed at 7DIV, indicating positive infection.

\section{In vitro optogenetic stimulation}

Thy1-ChR2 DRG neurons or cortical neurons infected with LV-ChR2 were used for in vitro optogenetic stimulation experiments. A 470nm emission LED array (LuxeonRebelTM) under the control of a Driver LED (FemtoBuck, SparkFun) of $600 \mathrm{~mA}$ and a pulse generator PulsePal (OpenEphys)(Siegle et al., 2017) was used to deliver blue light (470nm) to neuronal cultures. The 
117 optogenetic stimulation protocol consisted in $1 \mathrm{~h}$ of illumination at $20 \mathrm{~Hz}$ of frequency with $5 \mathrm{~ms}-45 \mathrm{~ms}$

118 pulses, in 1s ON-1s OFF periods. In the case of DRG neurons, stimulation was applied $2 \mathrm{~h}$ after seeding.

119 For cortical neurons, twodifferent stimulation time-points after the axotomy were assessed: $30 \mathrm{~min}$

120 after axotomy and 6h after axotomy.

\section{Corticospinal tract and dorsal columns axotomy}

A fine incision to the skin between the shoulder blades of anaesthetized mice and subsequent muscle removal allowed thoracic vertebral column exposure. A T9 laminectomy was performed, and the dura mater was excised. In corticospinal tract injuries, two thirds of the spinal cord approximately were severed laterally with a scalpel. In dorsal column axotomies (DCA), a dorsal hemisection was conducted with fine forceps.

\section{Sciatic nerve crush (SNC)}

128 The sciatic nerve was exposed after a small incision on the skin over the posterior hindlimb and blunt 129 dissection of the gluteus maximus and the biceps femoralis. Fine forceps were used to carefully 130 compress the nerve orthogonally $2 \times 10$ s. Animals were allowed to recover for $24 \mathrm{~h}$, when were then 131 sacrificed and the sciatic nerve and the sciatic DRGs (L4, L5, L6) were dissected and processed.

\section{In vivo optogenetic stimulation}

133 An optic fiber cannula (1.25mm, 0.22 NA; Thorlabs) was implanted in the motor cortex (M1) of Thy1$134 \mathrm{ChR} 2$ transgenic mice by stereotaxic surgery ( $-1 \mathrm{~mm}$ antero-posterior (AP), $1.5 \mathrm{~mm}$ lateral to bregma, $1350.5 \mathrm{~mm}$ deep, (hind limb innervation region)) prior to injury and was fixed to the cranium with screws 136 and dental cement. At 7 days post-injury (DPI) animals started receiving daily optogenetic stimulations 137 consisting in $1 \mathrm{~h}$ of illumination with $470 \mathrm{~nm}$ blue light, at $10 \mathrm{~Hz}$ of frequency with $10 \mathrm{~ms}$ pulses, in 138 1sON-1sOFF periods, for 5 consecutive days, 2 resting days followed by 5 more days. The illumination was delivered through the optic fiber cannula which was coupled to a 470nm wavelength LED source (M470F3, Thorlabs) controlled by a pulse generator (Pulse Pal, Open ephys) (Siegle et al., 2017). The control group went through the same procedures than the experimental group without receiving illumination.

\section{In vivo chemogenetic stimulation}

144 The commercial AAV5-hSynhM3D(Gq)-mCherry or the control virus AAV5-hSyn-mCherry 145 (Addgene) were injected into the sciatic nerve of WT C57BL/6J mice ( $1 \mu \mathrm{L} / \mathrm{sciatic}$ nerve) $4-5$ weeks 146 before the experiment. For chemogenetic stimulation, the animals received two daily intraperitoneal 147 (i.p.) injections of 5mg/kg CNO (Tocris). After DCA, injections were delivered starting from 3DPI and 148 lasted 7 consecutive days. In the case of SNC, the injections were given prior to injury: from 3 days pre-injury to the same day of the injury (in total 4 days of chemogenetic stimulation).

\section{Behavioral assessment of sensorimotor function}

Sensorimotor deficits and recovery were evaluated using the gridwalk and the BMS (Basso Mouse Scale) tests at $-1,1,7,14,21,28$ and 35 DPI for CST injuries and at $-1,1,3,7,10,21$ and 28 DPI for DCA. During the gridwalk animals were recorded while walking three times on a grid of $1 \mathrm{x} 1 \mathrm{~cm}$ squares (total longitude of the grid: $50 \mathrm{~cm}$ ). The number of missteps in relation to the total number of steps was blindly quantified. In BMS evaluations mice were allowed to freely move in an open field 
157 scale grades from 0 to 9 the locomotor capacity of the hind limbs depending on several aspects such as

158 ankle movement, paw placing and position or stepping.

\section{Tracing of injured spinal cords}

160 A fluorescent tracer (10\% Dextran AlexaFluor 594, ThermoFisher Scientific) was injected with a 161 stereotaxic frame (KOPF) into the motor cortex of animals with injured CST at 35DPI using the fibre optic cannula hole, in order to trace the stimulated CST. The tracer was injected at $0.2 \mu \mathrm{l} / \mathrm{minute}$ for a total of $1 \mu \mathrm{l}$, adding 5 more minutes at the end to avoid liquid spillage. $5 \mathrm{~d}$ were waited before sacrifice, to allow the tracer to be reach the axonal terminations

\section{Immunocytochemistry (ICC)}

166 For immunocytochemistry (ICC), cells were fixed with 4\% paraformaldehyde (PFA) 15 min on ice, 167 washed and incubated in blocking solution (1\% Bovine serum albumin (BSA), 0,25\% Triton X-100 $168(0,25 \% \mathrm{Tx})$ in PBS $(0.1 \mathrm{M}))$ for $1 \mathrm{~h}$ at RT. Primary antibody (anti- $\beta$ III tubulin (Tuj1, 1:1000, 169 BioLegend), c-Fos (1:200, Cell Signaling), ChR2 (1:500, Progen), GFP (1:500, ThermoFisher 170 Scientific) was added to the cells in blocking solution and incubated $\mathrm{O} / \mathrm{N}$ at $4^{\circ} \mathrm{C}$. After washing, the 171 cells were incubated $1 \mathrm{~h}$ at RT with Alexa-Fluor-conjugated secondary antibodies (568 goat and 488 172 goat and donkey).

\section{Tissue processing for immunohistochemistry}

174 Anaesthetized mice were transcardially perfused with ice-cold $4 \%$ paraformaldehyde (PFA) and the spinal cord, the brain or the DRGs were dissected and allowed to post-fix in PFA $4 \%$ for $24 \mathrm{~h}$ at $4^{\circ} \mathrm{C}$. In peripheral experiments the sciatic nerves and the DRGs were directly dissected, as perfusion was not needed, and allowed to fix in 4\% PFA for $2 \mathrm{~h}$ on ice. Fixed tissues were cryoprotected in $30 \%$ sucrose in PBS for $24 \mathrm{~h}$ at $4^{\circ} \mathrm{C}$, brains were then directly frozen and sliced at $30 \mu \mathrm{m}$ with a freezing microtome (2000R Leica), while spinal cords and DRGs were embedded in tissue freezing medium (OCT, Sigma Aldrich), frozen and $18 \mu \mathrm{m}$ or $10 \mu \mathrm{m}$ slices respectively were obtained using a cryostat (CM 1900 Leica) and directly mounted. For whole mount stainings cryprotection was not needed, instead tissues were kept in PBS.

\section{Immunohistochemistry (IHC)}

Prior to IHC, brain slices were maintained in cryoprotection solution (30\% glycerol, $30 \%$ ethylene glycol, 40\% PBS). Brain IHCs were performed directly on free floating slices. Slices were blocked for $1 \mathrm{~h}$ at RT $(10 \% \mathrm{FBS}, 0.5 \% \mathrm{Tx}, 0.2 \%$ gelatine, $0.2 \mathrm{M}$ glycine in $1 \mathrm{xPBS})$ and incubated with primary antibody $\mathrm{O} / \mathrm{N}$ at $4^{\circ} \mathrm{C}$ (5\% FBS, $0.5 \%$ Tx, $0.2 \%$ gelatine in PBS; GFP (1:500, ThermoFisher Scientific). Slices were then repeatedly washed with PBS-0.5\% Tx, incubated with secondary antibodies (Alexa Fluor 488 donkey) and Hoechst.

IHCs were performed directly on spinal cord preparation. Slides were blocked for $1 \mathrm{~h}$ at RT (Blocking solution: $8 \% \mathrm{BSA}, 0.3 \% \mathrm{Tx}$ in TBS) was added and incubated for $2 \mathrm{~h}$ at $\mathrm{RT}$, followed by O/N incubation 192 of primary antibody (GFAP (1:500, Dako) in 2\% BSA, $0.2 \%$ Tx in TBS at $4^{\circ} \mathrm{C}$. Secondary antibodies 193 (Alexa Fluor 488 donkey) were added after washing with TTBS and incubated 1h at RT. incubation (SCG-10 (1:1000, Novus Biologicals) in 2\% BSA, 0.3\% Tx in TBS at $4^{\circ} \mathrm{C}$. Secondary 
197 antibodies (Alexa Fluor 488 donkey) were added after washing with TTBS and incubated 1 O/N at

$1984^{\circ} \mathrm{C}$.

199 DRG preparations were stained with Hoechst (Sigma Aldrich).

200 Each preparation was subsequently mounted in Mowiol $^{\mathrm{TM}}$.

\section{Fluorescence intensity analysis}

202 To measure c-Fos intensity, DRG neurons were immunostained for c-Fos and ChR2 and imaged at 40x magnification with an Olympus microscope IX71 using an Orca Flash 4. Only ChR2 ${ }^{+}$cells were selected for this analysis. About 20 cells per well were analysed. The nuclei of the cells were selected and its mean c-FOS intensity determined by subtracting the corresponding background value to the integrated density (Corrected total cell fluorescence; CTCF), measured using Image ${ }^{\mathrm{TM}}$.

\section{Neurite and axonal length analysis}

Images were taken at 10x magnification with an Olympus microscope IX71 using an Orca Flash 4 or a CX50 Olympus camera. TUJ1 or GFP was used to immunolabel neurites and axons. For DRG neurite analysis, three fields per well were analysed and the average neurite length per cell was obtained. Small diameter $(<35 \mu \mathrm{m})$ neurons were excluded from the analysis. For cortical neuron cultures, 8-9 fields per device were analyzed. Either average axon length or total area covered by axons was quantified. Neurite-J plugin in Image $J^{\mathrm{TM}}$ software was used for neurite and axonal measurements (Meijering et al., 2004). Area measurements were measured using Image ${ }^{\mathrm{TM}}$ software.

\section{Sprouting quantification}

216 To quantify the number of sprouting axons before and after the lesion, we measured the tracer 217 fluorescence intensity on sections at $0,5 \mathrm{~mm}$ pre-injury and post-injury level. The spinal cord section 218 images were divided in two different ROIs corresponding to grey matter and white matter for analysis.

\section{Nerve regeneration analysis}

220 Regenerating sensory axons were immunolabeled with SCG10. Whole mount preparations were imaged with a confocal microscope (LSM 800, AxioCam 503c; Zeiss) at 5x magnification. 6-8 tiles and 10-15 slices were obtained per nerve and the $\operatorname{Zen}^{\mathrm{TM}}$ software and Image ${ }^{\mathrm{TM}}$ were used to reconstruct the nerve and obtain a Maximum Intensity Z-projection. The number of regenerating axons at several distances from the crush was determined.

\section{Statistical analysis}

226 Prism 6.0 (GraphPad ${ }^{\mathrm{TM}}$ Software) was used for statistics and graphical representation. Plotted data shows mean \pm s.e.m (standard error of the mean). Normality was determined with Shapiro-Wilk test. Significant differences are indicated by arterisks (* $p<0.05$; ** $p<0.01$; *** $p<0.005$; **** $p<0.001$ ). ANOVA followed by Bonferroni post hoc test or Student's t-test were used in normal distributions as opposed to Mann-Whitney or Kruskal-Wallis tests as non-parametric tests for samples that did not meet normality.

\section{$2323 \quad$ Results}




\section{Optogenetic stimulation of DRG neurons increase their axonal growth in vitro}

234 We first sought to determine if optogenetic activation of adult DRG neurons could enhance axonal growth in vitro. Adult dissociated Thy1-ChR2 DRG neurons were optically stimulated and allowed to grow for $24 \mathrm{~h}$.

Optical stimulation (Fig 1A) effectively increased neuronal activity in DRG neurons, as displayed by the increased levels of c-Fos staining after stimulation $(49627 \pm 4373$ a.u. of nuclear CTCF intensity for stimulated DRGs versus $33897 \pm 3212$ a.u. of nuclear CTCF intensity for non-stimulated; $p=0.0058$ Student's T test) (Fig 1B-C)

Optically stimulated neurons showed increased neurite outgrowth $(4053 \pm 433.1 \mu \mathrm{m})$ after $24 \mathrm{~h}$ of culture

\section{Chemogenetic stimulation of DRG neurons improve their regenerative capacity after sciatic nerve crush}

We wanted to determine if the in vitro results in fact translated to enhanced axon regeneration in vivo after PNS injury. Due to the difficulty to apply light for long periods of time in awake animals in the DRGs, we switched the activity control tool to chemogenetics.

We first wanted to determine if DRGs were transduced in vivo. AAV5-hSyn-mCherry or AAV5-hSynhM3Dq-mCherry were carefully injected into the sciatic nerve and mCherry expression was examined 4-5 weeks later. Both vectors transduced DRG neurons with similar efficiency ( $35 \%$ of total DRG neurons; $\sim 65 \%$ of large diameter $(>35 \mu \mathrm{m})$ neurons. mCherry expression was mainly localized in the soma of large diameter DRG neurons (Fig 2A).

To assess the effects of neuronal activity on PNS regeneration, animals received 2 daily injections of showed increased number of regenerating sensory axons $\left(\mathrm{SCG}^{+}\right)$at further distances $(>750 \mu \mathrm{m})$ when compared to mCherry controls. The two-way ANOVA showed a significant effect of the distance $(p<$ $0.0001)$ and stimulation $(p=0.0145)$ as well as their interaction $(p=0.0101)$. At $750 \mu \mathrm{m}$ from the injury stimulated animals showed $27.67 \pm 4.19$ axons versus $12.78 \pm 3.34$ axons on non-stimulated animals ( $p=0.0694$ Bonferroni post Hoc test), at $1000 \mu \mathrm{m}$ from the injury stimulated animals showed $21.56 \pm 3.72$ axons versus $6.44 \pm 1.43$ axons on non-stimulated animals $(p=0.0163$ Bonferroni post Hoc test), at $1250 \mu \mathrm{m}$ from the injury stimulated animals showed $14.89 \pm 3.12$ axons versus $3.11 \pm 1.14$ axons on non-stimulated animals ( $p=0.0261$ Bonferroni post Hoc test), and at $1500 \mu \mathrm{m}$ from the injury stimulated animals showed $8.56 \pm 1.81$ axons versus $1.56 \pm 1.06$ axons on non-stimulated animals ( $p=0.0259$ Bonferroni post Hoc test) (Fig 2 D-E).

\section{Optogenetic stimulation of cortical neurons does not improve the sensorimotor performance after} SCI

We then wanted to test if this increased regeneration capacity also was present in the CNS neurons. To this aim we first evaluated regenerative capacity of cortical neurons in vitro after optogenetic stimulation. Embryonic cortical neurons were seeded in custom microfluidic devices as previously described, and ChR2 was induced by infection with LV-ChR2 (Supp fig 1A), then axons were allowed to grow for few days through the microchannels until they reached the other chamber, when a vacuum assisted axotomy was performed. Optogenetic stimulation of cortical neurons decreased the axonal regrowth when stimulated 30 minutes after the axotomy (Supp Fig 1B). In contrast, 6 hours after axotomy, optogenetic stimulation resulted in a non- significant ( $p=0.0759$; Student T-Test) increase in 
axonal regrowth when compared to non-stimulated ones (Supp Fig 1 C-E). To control the intrinsic effect of blue light on neuronal growth, we applied the light stimulation pattern on non-stimulated (GFP controls) embryonic cortical neurons and observed no difference in axonal growth (Supp. Fig

278 1D).

279 To test if this results further translated to a better motor performance after SCI in vivo, we implanted optic fibre cannulas on Thy1-ChR2 animals, that have the cortical expression of ChR2 predominantly concentrated in the layer V, where corticospinal motor projecting neurons lay (Supp. Fig 2). After recovery, animals were subjected to a CST axotomy, and optical stimulation was performed daily from day 7 after injury (Fig 3A). Stimulated animals did not show any improvement in their motor performance on the Gridwalk (Fig 3B) or the BMS (Fig 3C) when compared to non-stimulated controls.

For each test evaluated, the two-way ANOVA showed a significant effect of the time (BMS $p<0.0001$; Gridwalk $p<0.0001$ ) but neither from the stimulation (BMS $p=0.7201$; Gridwalk $p=0.1308$ ) nor from their interaction (BMS $p=0.9905$; Gridwalk $p=0.0707$ ). In all tests, non-significant changes were observed for each timepoint when compared stimulated versus non-stimulated mice (Bonferroni multiple comparisons).

These results indicate that most likely CNS neurons have the ability to increase their axonal growth capacity when stimulated, but there are inhibitory signals in the CNS injury that blocks this regeneration in vivo.

Chemogenetic stimulation of DRG neurons does not improve their sensorimotor performance after $S C I$

To further address this issue, we tested the effects of chemogenetic stimulation on DRG neurons after a dorsal hemisection in vivo. Similarly, to what was done for the PNS injury, AAV5-hSyn-mCherry or AAV5-hSyn-hM3Dq-mCherry were carefully injected into the sciatic nerve to induce its expression on the DRG. 4-5 weeks later animals were subjected to a dorsal hemisection, and 3 days after injury animals received 2 daily injections of CNO ( $5 \mathrm{mg} / \mathrm{kg}$ b.w.) for 7 days (Fig $3 \mathrm{D}$ ). As observed for the cortical stimulation, stimulated animals did not show any improvement in their motor performance on the Gridwalk (Fig 3B) or the BMS (Fig 3C) when compared to mCherry-veh, or mCherry-CNO controls. For each test evaluated, the two-way ANOVA showed a significant effect of the time (BMS $p<0.0001$; Gridwalk $p<0.0001$ ) but neither from the stimulation (BMS $p=0.2289$; Gridwalk $p=0.0694$ ) nor from their interaction (BMS $p=0.3614$; Gridwalk $p=0.0785$ ). In all tests, non-significant changes were observed for each timepoint when compared stimulated versus non-stimulated mice (Bonferroni multiple comparisons).

Highlighting again the presence of an inhibitory environment in the CNS injury that blocks the regeneration induced by neuronal stimulation.

310 Optogenetic stimulation of dorsal root ganglia neurons increase their axonal growth in vitro, only 311 on permissive substrates

312 To further assess the impact of CNS inhibitory signals in the activity-induced increased axonal growth,

313 we cultured adult DRG neurons in both permissive (Laminin) and inhibitory (CSPG) substrates and 314 subjected them to optogenetic stimulation. 
315 As previously observed (Fig 1D-E), optogenetic stimulation increased the neurite outgrowth in 316 permissive substrates $(3682 \pm 316.2 \mu \mathrm{m}$ for stimulated DRGs versus $2721 \pm 183.1 \mu \mathrm{m}$ for non-stimulated 317 DRGs; $p=0.0128$ Student's T test), but not on neurons seeded on CSPGs $(86.4 \pm 13.4 \mu \mathrm{m}$ for stimulated 318 DRGs versus $73.04 \pm 10.27 \mu \mathrm{m}$ for non-stimulated DRGs; $p=0.4381$ Student's $T$ test), where 319 optogenetic stimulation did not produce any change in neurite outgrowth (Fig4 A-B).

\section{Optogenetic stimulation of corticospinal motor neurons increases prelesion sprouting but does not induce regeneration across the lesion.}

322 Since activity dependant therapies have been shown to increase lateral sprouting and plasticity 323 (Engesser-Cesar et al., 2007a; Goldshmit et al., 2008a; Carmel et al., 2010b, 2013a; Carmel and Martin, 324 2014b; Sánchez-Ventura et al., 2021), we sought to investigate if our stimulation paradigm increased the sprouting of injured axons before the lesion. To this aim, we analysed the spinal cords of stimulated Thy1-ChR2 animals after tracing their corticospinal tracts. Interestingly, we found that optogenetic stimulated animals did show more axonal tracing in the gray matter (33.60 \pm 1.13 a.u. of CTCF intensity for stimulated animals versus $23.27 \pm 0.89$ a.u. of CTCF intensity on non-stimulated animals; $p=0.0015$ Student's $\mathrm{T}$ test) in segments before $(-0.5 \mathrm{~mm})$ the injury, but not beyond $(+0.5 \mathrm{~mm})$ the injury (14.61 \pm 1.21 a.u. of CTCF intensity for stimulated animals versus $12.80 \pm 0.47$ a.u. of CTCF intensity on non-stimulated animals; $p=0.30328$ Student's T test) (Fig 4C-E). These findings support once again that the positive effects of modulation of the activity on the axonal regrowth are inhibited by CNS inhibitory signals present in the injury.

\section{Discussion}

Neuronal activity-triggered plasticity is commonly recognized as the main component of recovery in current activity-based therapies (Carulli et al., 2011; Hogan et al., 2020), however, this is mainly built on therapies that use exercise or electrical stimulation to induce neuronal activity (as in (EngesserCesar et al., 2007a; Goldshmit et al., 2008a; Carmel et al., 2010b, 2013a; Carmel and Martin, 2014b; Sánchez-Ventura et al., 2021)). Even so, even though these approaches increase neuronal activity, they do so without cellular specificity, hindering the identification of underlying cellular and molecular mechanisms induced by neuronal activity itself.

Taking advantage of the cellular specificity of optogenetics and chemogenetics (Sternson and Roth, 2014; Deisseroth, 2015) we performed different experiments to assess the specific cellular effects of neuronal activity on axonal growth using different in vitro and in vivo neuronal models.

345 Consistent with previous works (Park et al., 2015a; Ward et al., 2016; Jaiswal and English, 2017; SalaJarque et al., 2020), we showed that increasing neuronal activity on DRG sensory neurons enhanced axonal growth in non-inhibitory in vitro and in vivo peripheral injury models. Additionally, and still accordingly to previous studies (Ward et al., 2018), we also demonstrated these effects in embryonic cortical cells in vitro, highlighting the presence of similar mechanisms among different neuronal types.

It is important to mention that before the achievement of these results, a fine tuning of the stimulation protocol was needed, as axonal growth capacities showed to be highly dependent on the timing after injury and the pulse frequency during the stimulation (data not shown), highlighting the fact that neuronal activity needs, not only to be stimulated, but to be finely regulated in order to promote the desired outcomes. This has also been emphasized in previous studies indicating activity-dependant effects on gene expression (Lee et al., 2017; Tyssowski et al., 2018; Miyasaka and Yamamoto, 2021) and plasticity (reviewed in (Fauth and Tetzlaff, 2016)). 
In accordance, little changes in the stimulation patterns might lead to opposed effects, therefore, we used optogenetics, whenever possible, as our method of choice to stimulate activity since it allowed us a higher temporal resolution (Rost et al., 2017). Nonetheless, the anatomical setting of the DRGs impeded the implantation of a permanent optic fiber canula, and therefore did not allow us to perform awake stimulations, as this would have compromised the wellbeing of the animals, therefore, we opted for a chemogenetic approach for in vivo DRG stimulation. Effectively, this method rendered similar results on neurons as those observed in vitro with optogenetics, resulting in enhanced regeneration. For this experiment we delivered the DREADD agonist before the injury (as a preconditioning), leading to similar effects as those observed in previous studies using enriched environment (Hutson et al., 2019) or electrical stimulation (Udina et al., 2008b; Goganau et al., 2018b; Senger et al., 2018) as a preconditioning.

Since our results have shown comparable regeneration outcomes than electrical stimulations (Al-Majed et al., 2000, 2004; Ahlborn et al., 2007), we believe that neuronal activity itself might be responsible, at least partially, of these effects on axonal growth.

However, when we tested this paradigm in an in vivo model of SCI, we found that optogenetic stimulation of spinal-projecting cortical neurons did not promote the expected functional recovery after SCI, contrarily to what other studies using electrical stimulation (Carmel et al., 2010a, 2013b; Carmel and Martin, 2014a), or exercise (Engesser-Cesar et al., 2007b; Goldshmit et al., 2008b; Loy et al., 2018a) showed.

W then focused on clarifying whether our model did not induce any kind of axonal regeneration, or it did, but was insufficient to promote recovery. In that sense, an in vivo CNS model of injury implies the presence of a number of factors absent in our previous experiments, including the presence of extrinsic inhibitory substrates for regeneration (Mahar and Cavalli, 2018; Bradbury and Burnside, 2019; Mesquida-Veny et al., 2021). Accordingly, when we performed further histological characterization together with complementing in vitro experiments, we found that stimulated neurons displayed increased axonal growth in permissive substrates (laminin), but not in growth inhibitory substrates (CSPGs), and stimulated neurons showed an increased amount of growth and sprouting in the rostral vicinity of the injury, especially in the grey matter of the SC, but not caudally across or beyond the injury. These data suggests that neuronal activity stimulates the axonal growth capacity of these neurons through a mechanism that cannot overcome the repressive signalling of growthinhibitory molecules, such as CSPGs. However, in vivo injured neurons start growing through uninjured areas of the SC, similarly to what is described in other studies with classical stimulation approaches (Carmel et al., 2010a, 2013b; Carmel and Martin, 2014a), where injured and uninjured axons grow axonal processes searching for spared intraspinal circuitry, these new connections will eventually, through neuronal plasticity, restore the injured connections, bypassing the injury (Courtine and Sofroniew, 2019a). Interestingly, classical non-specific stimulations such as epidural electrical stimulation or rehabilitation, lead to stimulation of intraspinal circuits, involving spinal interneurons and motoneurons, this stimulation in turn promotes the reorganization and activation of this circuitry (Wagner et al., 2018a). Whether the lack of these functional effects with our paradigm was due to a shorter stimulation period, or that it lacked the direct stimulation of this intraspinal circuitry and thus not inducing the plasticity needed, remains still unanswered. of inhibitory-substrates after cellular specific stimulation (Wu et al., 2020a). In this study, functional 400 recovery after SCI was only achieved after combining chemogenetic stimulation and ChABC 401 administration (a CSPG degrading enzyme) (Wu et al., 2020b), surprisingly, functional recovery was 
402

403

404

405

406

407

408

409

410

411

412

413

414

415

416

417

418

419

420

421

422

423

424

425

426

427

428

429

430

431

432

433

434

435

436

437

438

439

440

441

442

443

444

445

446

447 only achieved after 6 weeks of continuous combined treatment. In contrast to both this work and our results, both chemogenetic stimulation and visual stimulation induce functional regeneration after optic nerve crush, a CNS injury model (Lim et al., 2016). Importantly however, the expression of the different chemogenetic receptors in this study was induced by intravitreal injection of the vectors, meaning that retinal interneurons, including amacrine and bipolar cells, will most likely be expressing the channels and be subjected to stimulation upon agonist administration. This paradigm resembles to that of electrical stimulation, considering activation of this retinal interneurons might be inducing neuronal plasticity that facilitates functional recovery.

There is plenty of evidence that neuronal activity itself is key in promoting regeneration and recovery, however, the presence of growth inhibitory substrates in the injured CNS limits the success of activitybased therapies. In line with this, combining functional rehabilitation or exercise with CSPG degrading therapies (including ChABC (García-Alías et al., 2009; Wang et al., 2011a) or ADAMTS4 (Griffin et al., 2020) lead to synergistic effects, even when applied at chronic time-points (Wang et al., 2011b). These reinforce the concept that multifactorial therapies are the way to go in order to tackle the different aspects of the pathophysiology of SCIs (Griffin and Bradke, 2020).

Although our experiments did not demonstrate the presence of an activity-triggered transcriptional switch, more chronic stimulations could be leading to it, although there are no evidences about that yet. Parallelly, more local and transient cellular mechanisms might explain the growth differences observed in our paradigm stimulating activity specifically at a cellular level. For example, neuronal activity changes the excitability of neurons through reorganization of several ionic channels (Misonou et al., 2004; Enes et al., 2010a; Romer et al., 2016). This can in turn influence growth, as a result of intracellular ionic adjustments and their downstream associated signalling (Shim and Ming, 2010; Tedeschi et al., 2016), or even translational changes (Enes et al., 2010b). Neuronal activity can also alter neuronal metabolism, increasing glycolysis, and lipid synthesis and integration to the membrane, a cellular process essential during axonal extension (Bas-Orth et al., 2017; Segarra-Mondejar et al., 2018). Another important cellular component altered by activity is the cytoskeleton and its dynamics, for instance, neuronal activity has been shown to increase dendritic spine microtubule polymerization (Hu et al., 2008). Accordingly, chemogenetic stimulation increases microtubule dynamics in the distal axonal portion, by reducing tubulin acetylation and increasing tyrosination in this region (Wu et al., 2020b). These mechanisms may not be exclusive, on the contrary, they are likely to take place all together facilitating activity-induced axonal growth.

Success of activity-based therapies does not depend solely on the cellular effects of activity modulations, instead, these therapies, that target activity in a more general and chronic way (as different neurons or even circuits are stimulated simultaneously), benefit from a raised general excitability that seems to be ultimate the responsible for the plasticity and reorganization that leads to recovery (Courtine and Sofroniew, 2019b). In that direction, rehabilitation and electrical stimulation after injury promote the formation of new synapses in the spinal cord (Loy et al., 2018b; Eisdorfer et al., 2020), respiratory function recovery after optogenetic stimulation is also attributed to synaptic plasticity (Alilain et al., 2008), and a recent study showing that rehabilitation together with electrochemical stimulation promotes recovery, is credited to cortico-reticulo-spinal circuit remodelling (Asboth et al., 2018). Additionally, sprouting of spared axons, instead of injured ones, is also responsible for recovery after exercise and/or electrical stimulation (Engesser-Cesar et al., 2007b; Goldshmit et al., 2008b; Carmel et al., 2010a, 2013b; Carmel and Martin, 2014a). In agreement to that, success of these approaches is only significant in incomplete injuries, and greater as more tissue is spared. Incomplete injuries might also help explaining why in our model we did not observe recovery after optogenetic stimulation of the motor cortex while others did (Deng et al., 2021), as different spinal injuries were 
used. Compellingly, compression injury leaves more uninjured tissue and spinal tracts than our injury, that axotomizes all the dorsal tracts, including the main component of the CST in mice. Besides, complete injuries also present larger glial scars, and therefore greater accumulation of growth inhibitory molecules, which translates in larger distances and hurdles to overcome or bypass to achieve functional connections, together with a greater loss of intraspinal circuits.

These observations strengthen the view that current activity-based therapies stimulate plasticity on top of inducing regeneration in specifically stimulated neurons, but probably without inducing long-lasting cellular reprogramming. This plasticity results from activity-triggered local changes, therefore prolonged stimulations are more effective increasing growth or regeneration (Courtine and Sofroniew, 2019a). This is also evidenced by the presence of functional recovery after 12, but not after 4 weeks of chemogenetic stimulation (Wu et al., 2020b). Therapeutically, holistic more unspecific approaches, have a more potent effect than cellular specific stimulations alone, as evidenced by enriched environment compared to chemogenetic stimulation, which presents a lower rate of regeneration (Hutson et al., 2019). Studies have also shown that this activity-induced plasticity can be accelerated and improved by linking the activation of different relays topographically in a system (McPherson et al., 2015; Mishra et al., 2017; Wagner et al., 2018b). These systems are however more challenging to define underlying mechanisms and understand the true nature of the gains of these therapies.

Briefly, we found that specific cellular stimulation of neuronal activity induced axonal growth, but only in the absence of inhibitory substrates. Likewise, this approach seems to be therapeutically less efficient in enhancing recovery than other more chronic or general stimulations. This also suggests and strengthens the idea that activity-based therapies succeed because of local transient cellular changes coupled with neuronal plasticity, rather than resulting in a cellular reprogramming of growth capacities, and that longer stimulation periods elicit more robust responses.

\section{$5 \quad$ Conflict of Interest}

472 The authors declare that the research was conducted in the absence of any commercial or financial relationships that could be construed as a potential conflict of interest.

\section{Author Contributions}

FMV performed, designed experiments, performed data analysis, and wrote the manuscript; SMT performed and designed experiments and performed data analysis; JADR supervised experiments, provided experimental funds and edited the manuscript; $\mathrm{AH}$ performed, designed experiments, performed data analysis, provided experimental funds and wrote the manuscript.

\section{$7 \quad$ Funding}

This research was supported by HDAC3-EAE-SCI Project with ref. PID2020-119769RA-I00 from MCIN/ AEI /10.13039/501100011033 to AH and PRPSEM Project with ref. RTI2018-099773-B-I00 from MCINN/AEI/10.13039/501100011033/ FEDER "Una manera de hacer Europa", the CERCA Programme, and the Commission for Universities and Research of the Department of Innovation, Universities, and Enterprise of the Generalitat de Catalunya (SGR2017-648) to JADR. The project leading to these results received funding from the María de Maeztu Unit of Excellence (Institute of Neurosciences, University of Barcelona) MDM-2017-0729 and Severo Ochoa Unit of Excellence (Institute of Bioengineering of Catalonia) CEX2018-000789-S from MCIN/AEI /10.13039/501100011033. FMV was supported by a fellowship from the "Ayudas para la Formación 


\section{Acknowledgments}

491 The authors thank Miriam Segura-Feliu and Ana Lopez-Mengual for their technical help.

492

493

494

495

496

497

498

499

500

501

502

503

504

505

506

507

508

509

510

511

512

513

514

515

516

517

518

519

520

521

522

523

524

525

\section{References}

Ahlborn, P., Schachner, M., and Irintchev, A. (2007). One hour electrical stimulation accelerates functional recovery after femoral nerve repair. Experimental Neurology 208, 137-144. doi:10.1016/j.expneurol.2007.08.005.

Alilain, W. J., Li, X., Horn, K. P., Dhingra, R., Dick, T. E., Herlitze, S., et al. (2008). Light-induced rescue of breathing after spinal cord injury. Journal of Neuroscience 28, 11862-11870. doi:10.1523/JNEUROSCI.3378-08.2008.

Al-Majed, A. A., Brushart, T. M., and Gordon, T. (2000). Electrical stimulation accelerates and increases expression of $\mathrm{BDNF}$ and trkB mRNA in regenerating rat femoral motoneurons. The European journal of neuroscience 12, 4381-4390.

Al-Majed, A. A., Siu, L. T., and Gordon, T. (2004). Electrical stimulation accelerates and enhances expression of regeneration-associated genes in regenerating rat femoral motoneurons. Cellular and Molecular Neurobiology 24, 379-402. doi:10.1023/B:CEMN.0000022770.66463.f7.

Anderson, M. A., O’Shea, T. M., Burda, J. E., Ao, Y., Barlatey, S. L., Bernstein, A. M., et al. (2018). Required growth facilitators propel axon regeneration across complete spinal cord injury. Nature 561. doi:10.1038/s41586-018-0467-6.

Arenkiel, B. R., Peca, J., Davison, I. G., Feliciano, C., Deisseroth, K., Augustine, G. J. J., et al. (2007). In Vivo Light-Induced Activation of Neural Circuitry in Transgenic Mice Expressing Channelrhodopsin-2. Neuron 54. doi:10.1016/j.neuron.2007.03.005.

Arlotta, P., Molyneaux, B. J., Chen, J., Inoue, J., Kominami, R., and MacKlis, J. D. (2005). Neuronal subtype-specific genes that control corticospinal motor neuron development in vivo. Neuron 45, 207-221. doi:10.1016/j.neuron.2004.12.036.

Asboth, L., Friedli, L., Beauparlant, J., Martinez-Gonzalez, C., Anil, S., Rey, E., et al. (2018). Cortico-reticulo-spinal circuit reorganization enables functional recovery after severe spinal cord contusion. Nature neuroscience 21, 576-588.

Bas-Orth, C., Tan, Y. W., Lau, D., and Bading, H. (2017). Synaptic activity drives a genomic program that promotes a neuronal warburg effect. Journal of Biological Chemistry 292, 51835194. doi:10.1074/jbc.M116.761106.

Basso, D. M., Fisher, L. C., Anderson, A. J., Jakeman, L. B., McTigue, D. M., and Popovich, P. G. (2006). Basso mouse scale for locomotion detects differences in recovery after spinal cord injury in five common mouse strains. Journal of Neurotrauma 23. doi:10.1089/neu.2006.23.635.

Bradbury, E. J., and Burnside, E. R. (2019). Moving beyond the glial scar for spinal cord repair. Nature Communications. doi:10.1038/s41467-019-11707-7. 
526

527

528

529

530

531

532

533

534

535

536

537

538

539

540

541

542

543

544

545

546

547

548

549

550

551

552

553

554

555

556

557

558

559

560

561

562

563

564

Carmel, J. B., Berrol, L. J., Brus-Ramer, M., and Martin, J. H. (2010a). Chronic electrical stimulation of the intact corticospinal system after unilateral injury restores skilled locomotor control and promotes spinal axon outgrowth. Journal of Neuroscience 30, 10918-10926. doi:10.1523/JNEUROSCI.1435-10.2010.

Carmel, J. B., Berrol, L. J., Brus-Ramer, M., and Martin, J. H. (2010b). Chronic electrical stimulation of the intact corticospinal system after unilateral injury restores skilled locomotor control and promotes spinal axon outgrowth. Journal of Neuroscience 30, 10918-10926. doi:10.1523/JNEUROSCI.1435-10.2010.

Carmel, J. B., Kimura, H., Berrol, L. J., and Martin, J. H. (2013a). Motor cortex electrical stimulation promotes axon outgrowth to brain stem and spinal targets that control the forelimb impaired by unilateral corticospinal injury. European Journal of Neuroscience 37, 1090-1102. doi:10.1111/ejn.12119.

Carmel, J. B., Kimura, H., Berrol, L. J., and Martin, J. H. (2013b). Motor cortex electrical stimulation promotes axon outgrowth to brain stem and spinal targets that control the forelimb impaired by unilateral corticospinal injury. European Journal of Neuroscience 37, 1090-1102. doi:10.1111/ejn.12119.

Carmel, J. B., Kimura, H., and Martin, J. H. (2014). Electrical stimulation of motor cortex in the uninjured hemisphere after chronic unilateral injury promotes recovery of skilled locomotion through ipsilateral control. Journal of Neuroscience 34, 462-466. doi:10.1523/JNEUROSCI.3315-13.2014.

Carmel, J. B., and Martin, J. H. (2014a). Motor cortex electrical stimulation augments sprouting of the corticospinal tract and promotes recovery of motor function. Frontiers in Integrative Neuroscience 8, 1-11. doi:10.3389/fnint.2014.00051.

Carmel, J. B., and Martin, J. H. (2014b). Motor cortex electrical stimulation augments sprouting of the corticospinal tract and promotes recovery of motor function. Frontiers in Integrative Neuroscience 8, 1-11. doi:10.3389/fnint.2014.00051.

Carulli, D., Foscarin, S., and Rossi, F. (2011). Activity-Dependent Plasticity and Gene Expression Modifications in the Adult CNS. Frontiers in Molecular Neuroscience 4, 1-12. doi:10.3389/fnmol.2011.00050.

Courtine, G., and Sofroniew, M. V. (2019a). Spinal cord repair: advances in biology and technology. Nature Medicine 25. doi:10.1038/s41591-019-0475-6.

Courtine, G., and Sofroniew, M. V. (2019b). Spinal cord repair: advances in biology and technology. Nature Medicine 25. doi:10.1038/s41591-019-0475-6.

Cregg, J. M., DePaul, M. A., Filous, A. R., Lang, B. T., Tran, A., and Silver, J. (2014). Functional regeneration beyond the glial scar. Experimental Neurology.

doi:10.1016/j.expneurol.2013.12.024.

Curcio, M., and Bradke, F. (2018). Axon Regeneration in the Central Nervous System: Facing the Challenges from the Inside. Annual Review of Cell and Developmental Biology. doi:10.1146/annurev-cellbio-100617-062508. 
565

566

567

568

569

570

571

572

573

574

575

576

577

578

579

580

581

582

583

584

585

586

587

588

589

590

591

592

593

594

595

596

597

598

599

600

601

602

Deisseroth, K. (2015). H I S TO R I C A L C O M M E N TA RY Optogenetics : 10 years of microbial opsins in neuroscience. 18, 1213-1225.

Deng, W. W., Wu, G. Y., Min, L. X., Feng, Z., Chen, H., Tan, M. L., et al. (2021). Optogenetic Neuronal Stimulation Promotes Functional Recovery After Spinal Cord Injury. Frontiers in Neuroscience 15, 1-10. doi:10.3389/fnins.2021.640255.

Eisdorfer, J. T., Smit, R. D., Keefe, K. M., Lemay, M. A., Smith, G. M., and Spence, A. J. (2020). Epidural Electrical Stimulation: A Review of Plasticity Mechanisms That Are Hypothesized to Underlie Enhanced Recovery From Spinal Cord Injury With Stimulation. Frontiers in Molecular Neuroscience 13, 1-12. doi:10.3389/fnmol.2020.00163.

Enes, J., Langwieser, N., Ruschel, J., Carballosa-Gonzalez, M. M., Klug, A., Traut, M. H., et al. (2010a). Electrical activity suppresses axon growth through Cav1.2 channels in adult primary sensory neurons. Current Biology 20, 1154-1164. doi:10.1016/j.cub.2010.05.055.

Enes, J., Langwieser, N., Ruschel, J., Carballosa-Gonzalez, M. M., Klug, A., Traut, M. H., et al. (2010b). Electrical activity suppresses axon growth through Cav1.2 channels in adult primary sensory neurons. Current Biology 20, 1154-1164. doi:10.1016/j.cub.2010.05.055.

Engesser-Cesar, C., Ichiyama, R. M., Nefas, A. L., Hill, M. A., Edgerton, V. R., Cotman, C. W., et al. (2007a). Wheel running following spinal cord injury improves locomotor recovery and stimulates serotonergic fiber growth. The European journal of neuroscience 25, 1931-1939.

Engesser-Cesar, C., Ichiyama, R. M., Nefas, A. L., Hill, M. A., Edgerton, V. R., Cotman, C. W., et al. (2007b). Wheel running following spinal cord injury improves locomotor recovery and stimulates serotonergic fiber growth. The European journal of neuroscience 25, 1931-1939.

Fauth, M., and Tetzlaff, C. (2016). Opposing effects of neuronal activity on structural plasticity. Frontiers in Neuroanatomy 10, 1-18. doi:10.3389/fnana.2016.00075.

Fitch, M. T., and Silver, J. (2008). CNS injury, glial scars, and inflammation: Inhibitory extracellular matrices and regeneration failure. Experimental Neurology. doi:10.1016/j.expneurol.2007.05.014.

Friel, K. M., Williams, P. T. J. A., Serradj, N., Chakrabarty, S., and Martin, J. H. (2014). ActivityBased Therapies for Repair of the Corticospinal System Injured during Development. Frontiers in Neurology 5. doi:10.3389/fneur.2014.00229.

García-Alías, G., Barkhuysen, S., Buckle, M., and Fawcett, J. W. (2009). Chondroitinase ABC treatment opens a window of opportunity for task-specific rehabilitation. Nature Neuroscience 12, 1145-1151. doi:10.1038/nn.2377.

Goganau, I., Sandner, B., Weidner, N., Fouad, K., and Blesch, A. (2018a). Depolarization and electrical stimulation enhance in vitro and in vivo sensory axon growth after spinal cord injury. Experimental Neurology 300, 247-258. doi:10.1016/j.expneurol.2017.11.011.

Goganau, I., Sandner, B., Weidner, N., Fouad, K., and Blesch, A. (2018b). Depolarization and electrical stimulation enhance in vitro and in vivo sensory axon growth after spinal cord injury. Experimental Neurology 300, 247-258. doi:10.1016/j.expneurol.2017.11.011. 
603

604

605

606

607

608

609

610

611

612

613

614

615

616

617

618

619

620

621

622

623

624

625

626

627

628

629

630

631

632

633

634

635

636

637

638

Goldshmit, Y., Lythgo, N., Galea, M. P., and Turnley, A. M. (2008a). Treadmill training after spinal cord hemisection in mice promotes axonal sprouting and synapse formation and improves motor recovery. Journal of Neurotrauma 25, 449-465. doi:10.1089/neu.2007.0392.

Goldshmit, Y., Lythgo, N., Galea, M. P., and Turnley, A. M. (2008b). Treadmill training after spinal cord hemisection in mice promotes axonal sprouting and synapse formation and improves motor recovery. Journal of Neurotrauma 25, 449-465. doi:10.1089/neu.2007.0392.

Griffin, J. M., and Bradke, F. (2020). Therapeutic repair for spinal cord injury: combinatory approaches to address a multifaceted problem. EMBO Molecular Medicine 12, 1-29. doi:10.15252/emmm.201911505.

Griffin, J. M., Fackelmeier, B., Clemett, C. A., Fong, D. M., Mouravlev, A., Young, D., et al. (2020). Astrocyte-selective AAV-ADAMTS4 gene therapy combined with hindlimb rehabilitation promotes functional recovery after spinal cord injury. Experimental Neurology 327. doi:10.1016/j.expneurol.2020.113232.

He, Z., and Jin, Y. (2016). Intrinsic Control of Axon Regeneration. Neuron. doi:10.1016/j.neuron.2016.04.022.

Hogan, M. K., Hamilton, G. F., and Horner, P. J. (2020). Neural Stimulation and Molecular Mechanisms of Plasticity and Regeneration: A Review. Frontiers in Cellular Neuroscience 14, 1-16. doi:10.3389/fncel.2020.00271.

Hu, X., Viesselmann, C., Nam, S., Merriam, E., and Dent, E. W. (2008). Activity-dependent dynamic microtubule invasion of dendritic spines. Journal of Neuroscience 28, 13094-13105. doi:10.1523/JNEUROSCI.3074-08.2008.

Huebner, E. A., and Strittmatter, S. M. (2009). Axon regeneration in the peripheral and central nervous systems. Results and Problems in Cell Differentiation 48. doi:10.1007/400_2009_19.

Hutson, T. H., Kathe, C., Palmisano, I., Bartholdi, K., Hervera, A., de Virgiliis, F., et al. (2019). Cbpdependent histone acetylation mediates axon regeneration induced by environmental enrichment in rodent spinal cord injury models. Science Translational Medicine 11. doi:10.1126/scitranslmed.aaw2064.

Jaiswal, P. B., and English, A. W. (2017). Chemogenetic enhancement of functional recovery after a sciatic nerve injury. European Journal of Neuroscience 45, 1252-1257. doi:10.1111/ejn.13550.

Jones, L. L., Sajed, D., and Tuszynski, M. H. (2003). Axonal Regeneration through Regions of Chondroitin Sulfate Proteoglycan Deposition after Spinal Cord Injury: A Balance of Permissiveness and Inhibition. Journal of Neuroscience 23. doi:10.1523/jneurosci.23-2809276.2003.

Lee, P. R., Cohen, J. E., Iacobas, D. A., Iacobas, S., and Douglas Fields, R. (2017). Gene networks activated by specific patterns of action potentials in dorsal root ganglia neurons. Scientific Reports 7, 1-14. doi:10.1038/srep43765. 
639

640

641

642

643

644

645

646

647

648

649

650

651

652

653

654

655

656

657

658

659

660

661

662

663

664

665

666

667

668

669

670

671

672

673

674

675

Lim, J. H. A., Stafford, B. K., Nguyen, P. L., Lien, B. V., Wang, C., Zukor, K., et al. (2016). Neural activity promotes long-distance, target-specific regeneration of adult retinal axons. Nature Neuroscience 19, 1073-1084. doi:10.1038/nn.4340.

Loy, K., Schmalz, A., Hoche, T., Jacobi, A., Kreutzfeldt, M., Merkler, D., et al. (2018a). Enhanced Voluntary Exercise Improves Functional Recovery following Spinal Cord Injury by Impacting the Local Neuroglial Injury Response and Supporting the Rewiring of Supraspinal Circuits. Journal of neurotrauma 35, 294-2915.

Loy, K., Schmalz, A., Hoche, T., Jacobi, A., Kreutzfeldt, M., Merkler, D., et al. (2018b). Enhanced Voluntary Exercise Improves Functional Recovery following Spinal Cord Injury by Impacting the Local Neuroglial Injury Response and Supporting the Rewiring of Supraspinal Circuits. Journal of neurotrauma 35, 294-2915.

Mahar, M., and Cavalli, V. (2018). Intrinsic mechanisms of neuronal axon regeneration. Nature Reviews Neuroscience. doi:10.1038/s41583-018-0001-8.

McPherson, J. G., Miller, R. R., Perlmutter, S. I., and Poo, M. M. (2015). Targeted, activitydependent spinal stimulation produces long-lasting motor recovery in chronic cervical spinal cord injury. Proceedings of the National Academy of Sciences of the United States of America 112, 12193-12198. doi:10.1073/pnas.1505383112.

Meijering, E., Jacob, M., Sarria, J. C. F., Steiner, P., Hirling, H., and Unser, M. (2004). Design and Validation of a Tool for Neurite Tracing and Analysis in Fluorescence Microscopy Images. Cytometry Part A 58. doi:10.1002/cyto.a.20022.

Mesquida-Veny, F., del Río, J. A., and Hervera, A. (2021). Macrophagic and microglial complexity after neuronal injury. Progress in Neurobiology 200. doi:10.1016/j.pneurobio.2020.101970.

Mishra, A. M., Pal, A., Gupta, D., and Carmel, J. B. (2017). Paired motor cortex and cervical epidural electrical stimulation timed to converge in the spinal cord promotes lasting increases in motor responses. Journal of Physiology 595, 6953-6968. doi:10.1113/JP274663.

Misonou, H., Mohapatra, D. P., Park, E. W., Leung, V., Zhen, D., Misonou, K., et al. (2004). Regulation of ion channel localization and phosphorylation by neuronal activity. Nature Neuroscience 7, 711-718. doi:10.1038/nn1260.

Miyasaka, Y., and Yamamoto, N. (2021). Neuronal Activity Patterns Regulate Brain-Derived Neurotrophic Factor Expression in Cortical Cells via Neuronal Circuits. Frontiers in Neuroscience 15. doi:10.3389/fnins.2021.699583.

Ohtake, Y., and Li, S. (2015). Molecular mechanisms of scar-sourced axon growth inhibitors. Brain Research 1619. doi:10.1016/j.brainres.2014.08.064.

Park, S., Koppes, R. A., Froriep, U. P., Jia, X., Achyuta, A. K. H., McLaughlin, B. L., et al. (2015a). Optogenetic control of nerve growth. Scientific Reports 5, 1-9. doi:10.1038/srep09669.

Park, S., Koppes, R. A., Froriep, U. P., Jia, X., Achyuta, A. K. H., McLaughlin, B. L., et al. (2015b). Optogenetic control of nerve growth. Scientific Reports 5, 1-9. doi:10.1038/srep09669. 
676

677

678

679

680

681

682

683

684

685

686

687

688

689

690

691

692

693

694

695

696

697

698

699

700

701

702

703

704

705

706

707

708

709

710

711

712

Richardson, P. M., McGuinness, U. M., and Aguayo, A. J. (1980). Axons from CNS neurones regenerate into PNS grafts. Nature 284. doi:10.1038/284264a0.

Romer, S. H., Deardorff, A. S., and Fyffe, R. E. W. (2016). Activity-dependent redistribution of Kv2.1 ion channels on rat spinal motoneurons. Physiological Reports 4, 1-11. doi:10.14814/phy2.13039.

Rost, B. R., Schneider-warme, F., Schmitz, D., and Hegemann, P. (2017). Primer Optogenetic Tools for Subcellular Applications in Neuroscience. Neuron 96, 572-603. doi:10.1016/j.neuron.2017.09.047.

Sala-Jarque, J., Mesquida-Veny, F., Badiola-Mateos, M., Samitier, J., Hervera, A., and del Río, J. A. (2020). Neuromuscular Activity Induces Paracrine Signaling and Triggers Axonal Regrowth after Injury in Microfluidic Lab-On-Chip Devices. Cells 9, 302. doi:10.3390/cells9020302.

Sami, A., Selzer, M. E., and Li, S. (2020). Advances in the Signaling Pathways Downstream of GlialScar Axon Growth Inhibitors. Frontiers in Cellular Neuroscience 14. doi:10.3389/fncel.2020.00174.

Sánchez-Ventura, J., Giménez-Llort, L., Penas, C., and Udina, E. (2021). Voluntary wheel running preserves lumbar perineuronal nets, enhances motor functions and prevents hyperreflexia after spinal cord injury. Experimental Neurology 336. doi:10.1016/j.expneurol.2020.113533.

Segarra-Mondejar, M., Casellas-Díaz, S., Ramiro-Pareta, M., Müller-Sánchez, C., Martorell-Riera, A., Hermelo, I., et al. (2018). Synaptic activity-induced glycolysis facilitates membrane lipid provision and neurite outgrowth. The EMBO Journal 37, 1-16. doi:10.15252/embj.201797368.

Senger, J. L. B., Verge, V. M. K., Macandili, H. S. J., Olson, J. L., Chan, K. M., and Webber, C. A. (2018). Electrical stimulation as a conditioning strategy for promoting and accelerating peripheral nerve regeneration. Experimental Neurology 302, 75-84. doi:10.1016/j.expneurol.2017.12.013.

Shim, S., and Ming, G. L. (2010). Roles of channels and receptors in the growth cone during PNS axonal regeneration. Experimental Neurology 223, 38-44. doi:10.1016/j.expneurol.2009.10.001.

Siegle, J. H., López, A. C., Patel, Y. A., Abramov, K., Ohayon, S., and Voigts, J. (2017). Open Ephys: An open-source, plugin-based platform for multichannel electrophysiology. Journal of Neural Engineering 14. doi:10.1088/1741-2552/aa5eea.

Sternson, S. M., and Roth, B. L. (2014). Chemogenetic tools to interrogate brain functions. Annual Review of Neuroscience 37, 387-407. doi:10.1146/annurev-neuro-071013-014048.

Taylor, A. M., Blurton-Jones, M., Rhee, S. W., Cribbs, D. H., Cotman, C. W., and Jeon, N. L. (2005). A microfluidic culture platform for CNS axonal injury, regeneration and transport. Nature Methods 2. doi:10.1038/nmeth777.

Tedeschi, A., Dupraz, S., Laskowski, C. J., Xue, J., Ulas, T., Beyer, M., et al. (2016). The Calcium Channel Subunit Alpha2delta2 Suppresses Axon Regeneration in the Adult CNS. Neuron 92, 419-434. doi:10.1016/j.neuron.2016.09.026. 
713

714

715

716

717

718

719

720

721

722

723

724

725

726

727

728

729

730

731

732

733

734

735

736

737

738

739

740

741

742

743

744

745

746

747

748

749

750

Tyssowski, K. M., DeStefino, N. R., Cho, J. H., Dunn, C. J., Poston, R. G., Carty, C. E., et al. (2018). Different Neuronal Activity Patterns Induce Different Gene Expression Programs. Neuron 98, 530-546.e11. doi:10.1016/j.neuron.2018.04.001.

Udina, E., Furey, M., Busch, S., Silver, J., Gordon, T., and Fouad, K. (2008a). Electrical stimulation of intact peripheral sensory axons in rats promotes outgrowth of their central projections. Experimental Neurology 210, 238-247. doi:10.1016/j.expneurol.2007.11.007.

Udina, E., Furey, M., Busch, S., Silver, J., Gordon, T., and Fouad, K. (2008b). Electrical stimulation of intact peripheral sensory axons in rats promotes outgrowth of their central projections. Experimental Neurology 210, 238-247. doi:10.1016/j.expneurol.2007.11.007.

Wagner, F. B., Mignardot, J. B., Le Goff-Mignardot, C. G., Demesmaeker, R., Komi, S., Capogrosso, M., et al. (2018a). Targeted neurotechnology restores walking in humans with spinal cord injury. Nature 563, 65-93. doi:10.1038/s41586-018-0649-2.

Wagner, F. B., Mignardot, J. B., Le Goff-Mignardot, C. G., Demesmaeker, R., Komi, S., Capogrosso, M., et al. (2018b). Targeted neurotechnology restores walking in humans with spinal cord injury. Nature 563, 65-93. doi:10.1038/s41586-018-0649-2.

Wang, D., Ichiyama, R. M., Zhao, R., Andrews, M. R., and Fawcett, J. W. (2011a). Chondroitinase combined with rehabilitation promotes recovery of forelimb function in rats with chronic spinal cord injury. Journal of Neuroscience 31, 9332-9344. doi:10.1523/JNEUROSCI.0983-11.2011.

Wang, D., Ichiyama, R. M., Zhao, R., Andrews, M. R., and Fawcett, J. W. (2011b). Chondroitinase combined with rehabilitation promotes recovery of forelimb function in rats with chronic spinal cord injury. Journal of Neuroscience 31, 9332-9344. doi:10.1523/JNEUROSCI.0983-11.2011.

Ward, P. J., Clanton, S. L., and English, A. W. (2018). Optogenetically enhanced axon regeneration: motor versus sensory neuron-specific stimulation. European Journal of Neuroscience 47. doi:10.1111/ejn.13836.

Ward, P. J., Jones, L. N., Mulligan, A., Goolsby, W., Wilhelm, J. C., and English, A. W. (2016). Optically-induced neuronal activity is sufficient to promote functional motor axon regeneration in vivo. PLoS ONE 11, 1-16. doi:10.1371/journal.pone.0154243.

Wu, D., Jin, Y., Shapiro, T. M., Hinduja, A., Baas, P. W., and Tom, V. J. (2020a). Chronic neuronal activation increases dynamic microtubules to enhance functional axon regeneration after dorsal root crush injury. Nature Communications 11, 1-16. doi:10.1038/s41467-020-19914-3.

Wu, D., Jin, Y., Shapiro, T. M., Hinduja, A., Baas, P. W., and Tom, V. J. (2020b). Chronic neuronal activation increases dynamic microtubules to enhance functional axon regeneration after dorsal root crush injury. Nature Communications 11, 1-16. doi:10.1038/s41467-020-19914-3.

Wu, D., Klaw, M. C., Connors, T., Kholodilov, N., Burke, R. E., and Tom, V. J. (2015). Expressing constitutively active rheb in adult neurons after a complete spinal cord injury enhances axonal regeneration beyond a chondroitinase-treated glial scar. Journal of Neuroscience 35. doi:10.1523/JNEUROSCI.0719-15.2015. 


\section{Figure legends}

752

Figure 1. Increased neuronal activity promotes neurite outgrowth. A. Diagram of the optogenetic illumination protocol. B. Representative images of c-Fos (red) and ChR2 (green) immunostaining. Scale bar: $50 \mu \mathrm{m}$. C. c-Fos expression in the nucleus is increased just after optogenetic stimulation of Thy1-ChR2 DRG neurons ( $n=20-25$ cells). MFI: mean fluorescence intensity. a.u.: arbitrary units. Data are expressed as mean nuclear fluorescence intensity \pm s.e.m. ${ }^{* *} p$ $<0.01$ denotes significant differences in Student's t-test. D. Stimulated neurons presented significantly higher neurite lengths when compared to non-stimulated ones. Data are expressed as average neurite length per neuron \pm s.e.m $24 \mathrm{~h}$ in vitro $\left(\mathrm{n}=7\right.$ wells from 3 different experiments). ${ }^{*} p<$ 0.05 denotes significant differences in Student's t-test. E. Representative images of Tuj-1 staining used to analyze neurite length. Scale bar: $500 \mu \mathrm{m}$.

Figure 2. Chemogenetic stimulation induced PNS regeneration. A-B. mCherry expression in AAV-mCherry and AAV-hM3Dq infected DRG neurons 4-5 weeks after viral injection. Largediameter DRG neurons are preferentially infected. Data are expressed as mean \% of large-diameter (diameter $>35 \mu \mathrm{m})$ infected neurons \pm s.e.m. ( $\mathrm{n}=7-9$ DRGs per group). Scale bar: $100 \mu \mathrm{m}$. C. Schematic timeline of the experiment. D. The number of regenerating sensory axons (SCG-10 ${ }^{+}$) in stimulated nerves (hM3Dq-CNO) is increased in all assessed distances compared to the non-stimulated (mCherry-CNO) reaching statistical significance in long distances. Data are expressed as mean \pm s.e.m. at each distance from the injury site ( $\mathrm{n}=9$ sciatic nerves per group). $* p<0.05$ denotes significant differences in ANOVA followed by Bonferroni post-hoc test. E. SCG-10 immunostaining of mCherry or hM3Dq infected sciatic nerves $24 \mathrm{~h}$ after SNC. Dotted lines indicate the injury site. Scale bar: $200 \mu \mathrm{m}$

Figure 3. Increasing neuronal activity does not induce recovery after CNS injury. A. Timeline of the CST injury and stimulation experiments. B-C. \% of missteps in the gridwalk test (B) (n=8-9 mice per group for each timepoint) and BMS score $(C)(n=10$ mice per group for each timepoint) show no differences in sensorimotor recovery in stimulated Thy1-ChR2 (ChR2 Light) mice when compared to non-stimulated (ChR2 No light) after CST injury. D. Timeline of the DCA and stimulation experiments. E-F. Chemogenetically stimulated animals (hM3Dq-CNO) show similar sensorimotor function recovery to non-stimulated ones (mCherry-CNO, mCherry-veh) after DCA as seen by the gridwalk (E) (n=3-5 mice per group for each timepoint) and BMS (F) (n=5-6 mice per group for each timepoint) tests. Represented data correspond to the BMS score and \% of missteps in the gridwalk. Data are expressed as mean \pm s.e.m. ANOVA followed by Bonferroni post-hoctest.

\section{Figure 4. Neuronal activity induces growth but is not sufficient to overcome inhibitory} environments. A. Neurite outgrowth was significantly increased in stimulated DRG neurons on growth-permissive substrates (LAM: laminin), but not over non-permissive substrates (CSPGs). Average neurite length per neuron was determined at $24 \mathrm{~h}$ in vitro. Data are expressed as mean \pm s.e.m ( $\mathrm{n}=11-23$ images from 3 different experiments). ${ }^{*} p<0.05$ denotes significant differences in Student's t-test. B. Representative images of Tuj-1 staining used to analyze neurite length. Scale bar: $200 \mu \mathrm{m}$. C-D. Tracer intensity quantification of injured stimulated and non-stimulated Thy1-ChR2 mice at $0,5 \mathrm{~mm}$ pre-injury and post-injury. The spinal cord was divided in grey matter and white matter for analysis. Optogenetic stimulation of the CST resulted in increased sprouting in the grey matter pre-injury, but not post-injury. a.u.: arbitrary units. Data are expressed as mean tracer intensity \pm s.e.m ( $\mathrm{n}=5$ spinal cords per group). ${ }^{*} p<0.01$ denotes significant differences in Student's 
795 Supplementary Figure 1. Optogenetic stimulation of cortical neurons after axotomy. A. Cortical neurons (Tuj-1) express ChR2-eYFP after LV-ChR2 infection. White arrows in the high magnification image indicate neurite expression of ChR2. Scale bar: $250 \mu \mathrm{m}$. B-C. Optogenetic

798 stimulation of cortical neurons 30min after axotomy (B) resulted in reduced axon regeneration, while delivering the stimulation $6 \mathrm{~h}$ after axotomy $(\mathrm{C})$ increased axon regeneration without reaching

800 statistical significance. Individual ChR2 ${ }^{+}$axon lengths were quantified (B: $n=16$ images; $C: n=7-15$

801 images). Data are expressed as mean \pm s.e.m. ${ }^{* * *} p<0.001$ denotes significant differences in Student's 802 t-test. D. Optogenetic stimulation (ChR2 Light) of WT cortical neurons did not induce any changes 803 in axonal regeneration. Total growth area/microchannel was computed $(n=7-8$ images corresponding 804 to 4 different devices). Data are expressed as mean \pm s.e.m. E. Representative images of GFP staining 805 used to analyze axon regeneration when stimulation is applied $6 \mathrm{~h}$ after axotomy. Scale bar: $200 \mu \mathrm{m}$.

806 Supplementary Figure 2. ChR2 expression in neocortex of Thy1-ChR2 mice. While some layer II-III neurons express ChR2, expression is concentrated in layer V neurons. Scale bar: $100 \mu \mathrm{m}$ 


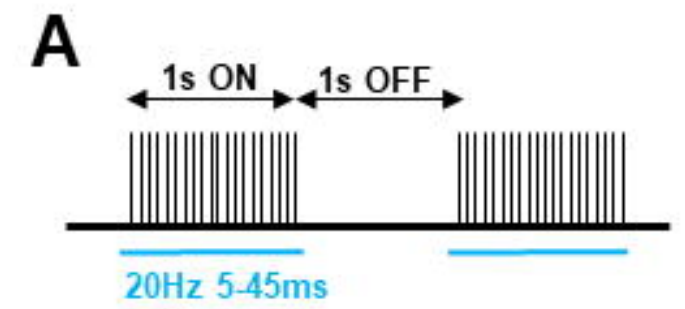

C

B

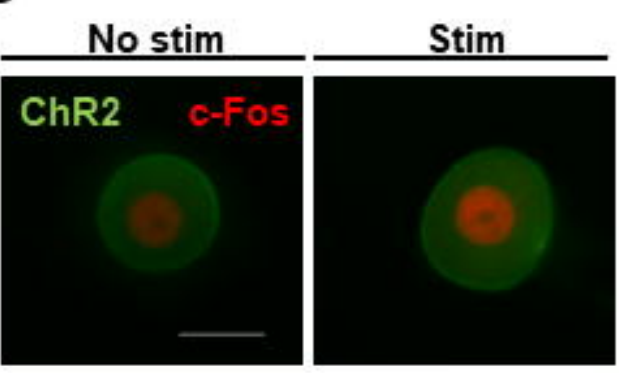

D
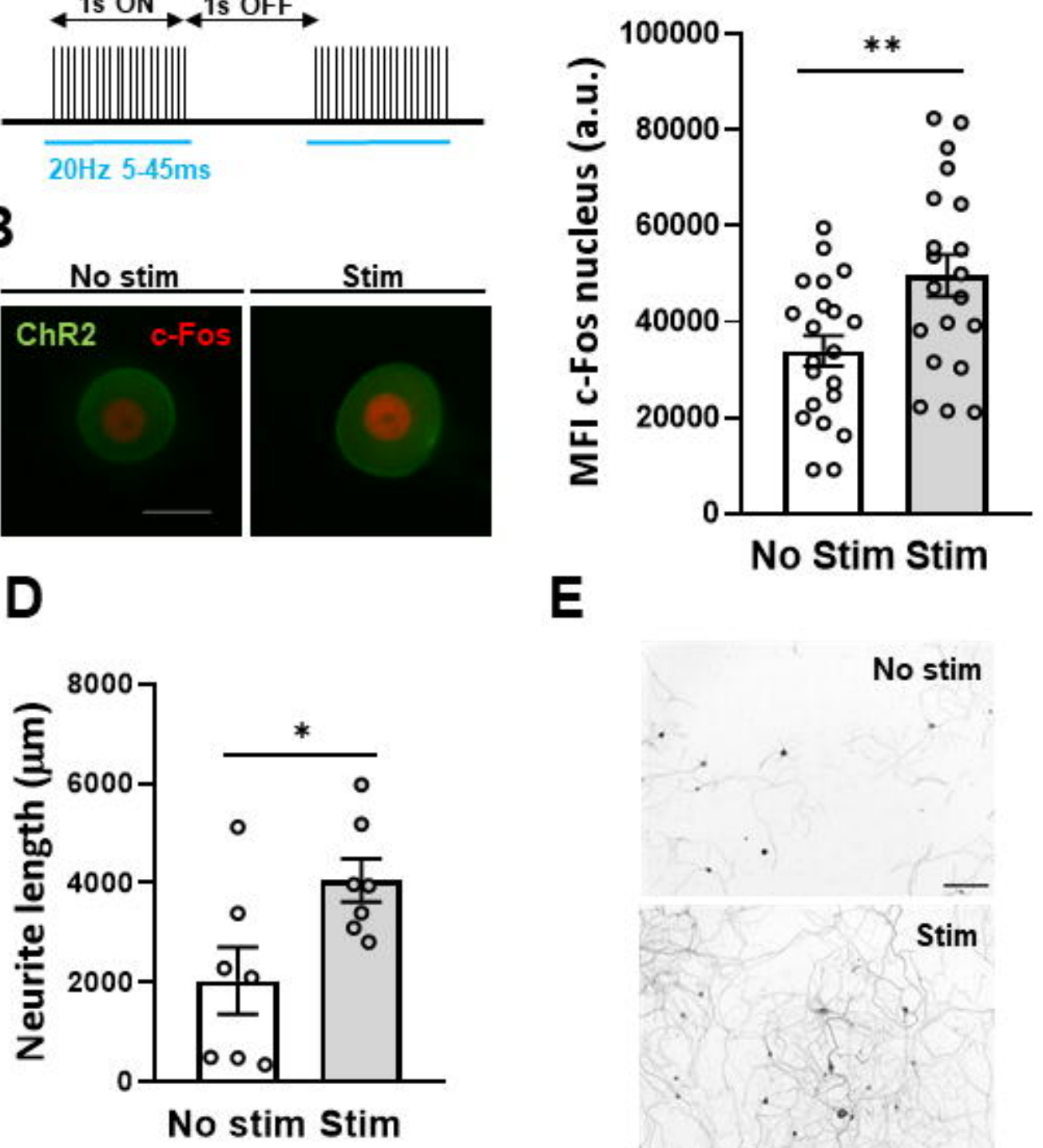

No stim

Stim

Figure 1 

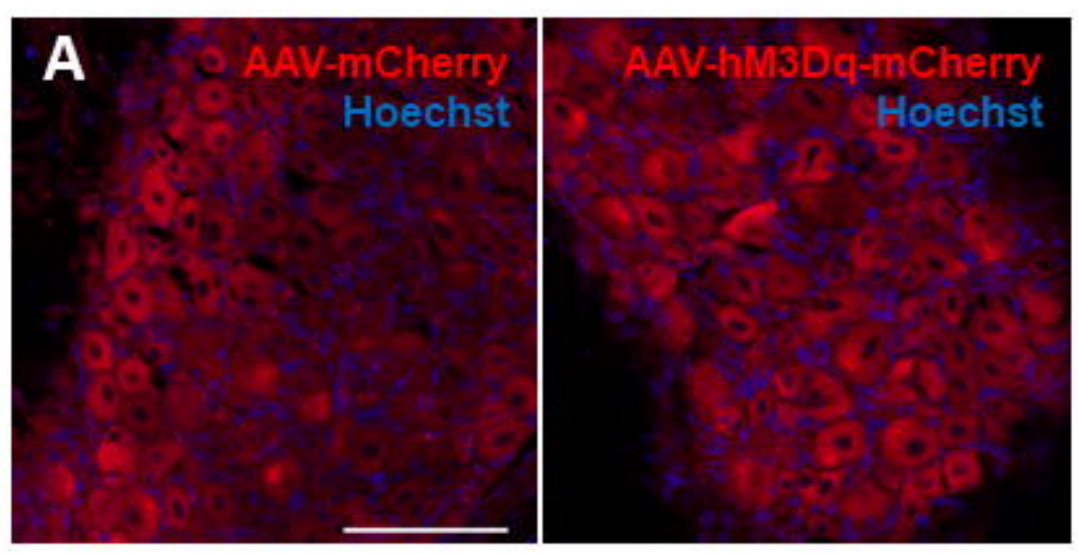

B

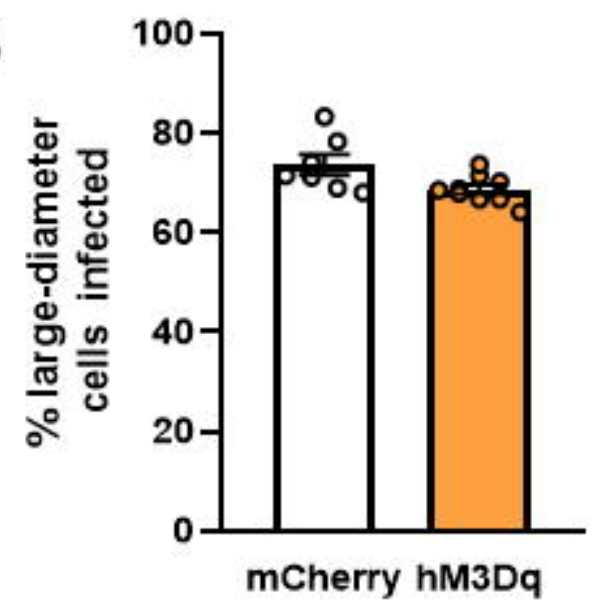

E
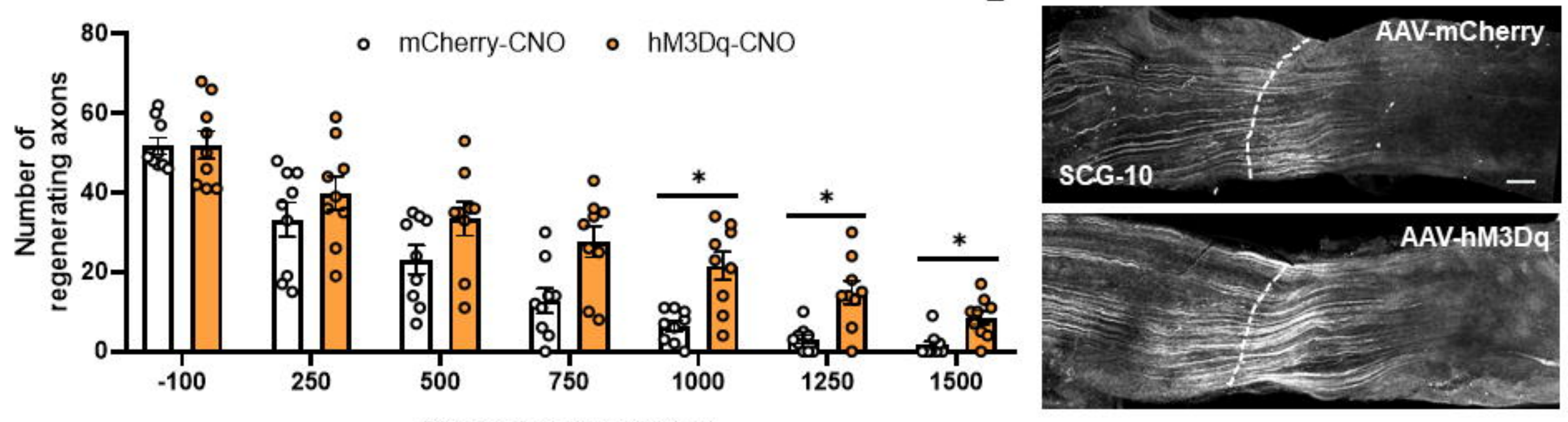

Distance from injury site ( $\mu \mathrm{m})$ 
A

Optic fiber

impantation

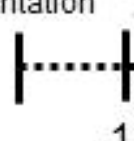

B
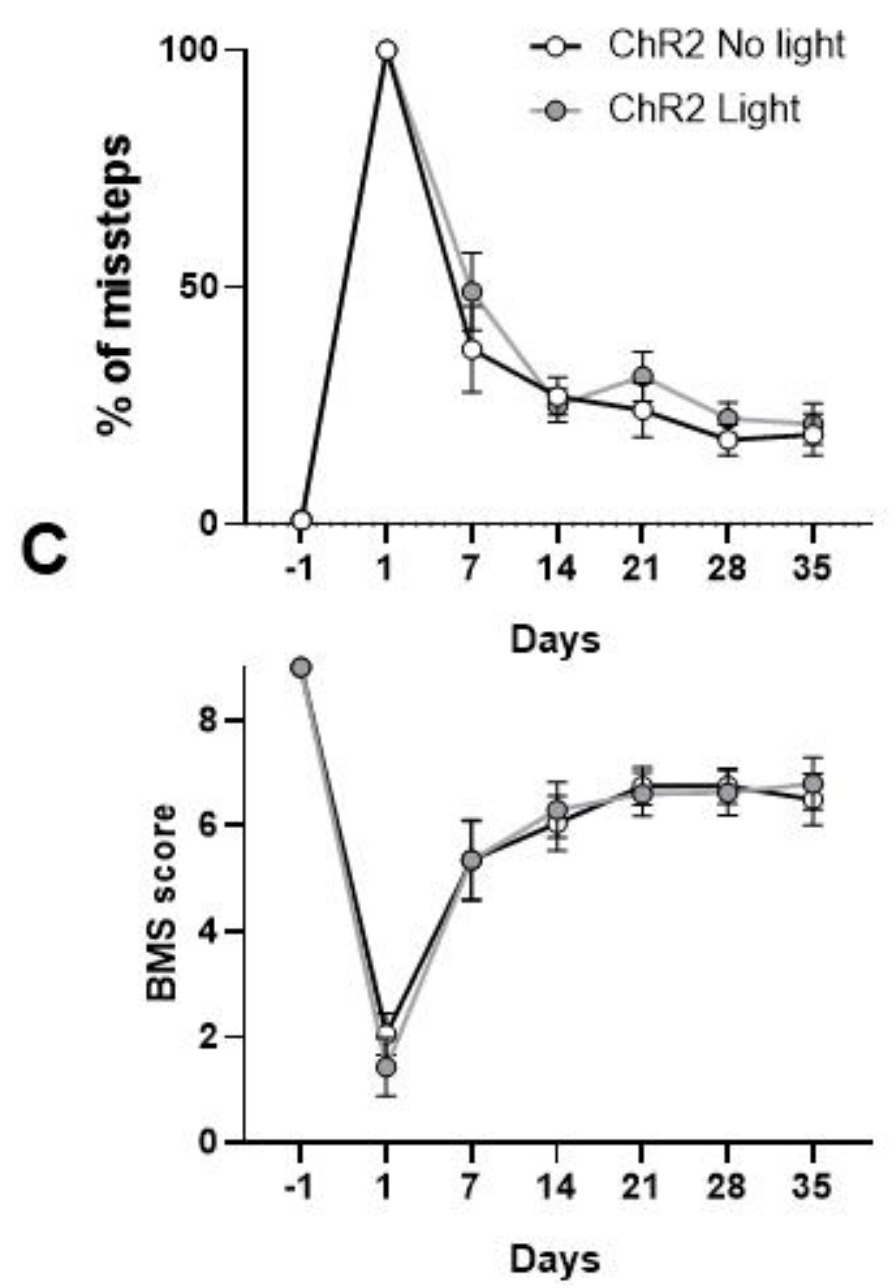

D

AAV

injection

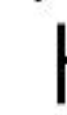

weeks

BMS/Gridwalk

Perfusion

$-4-5$ weeks

E

F
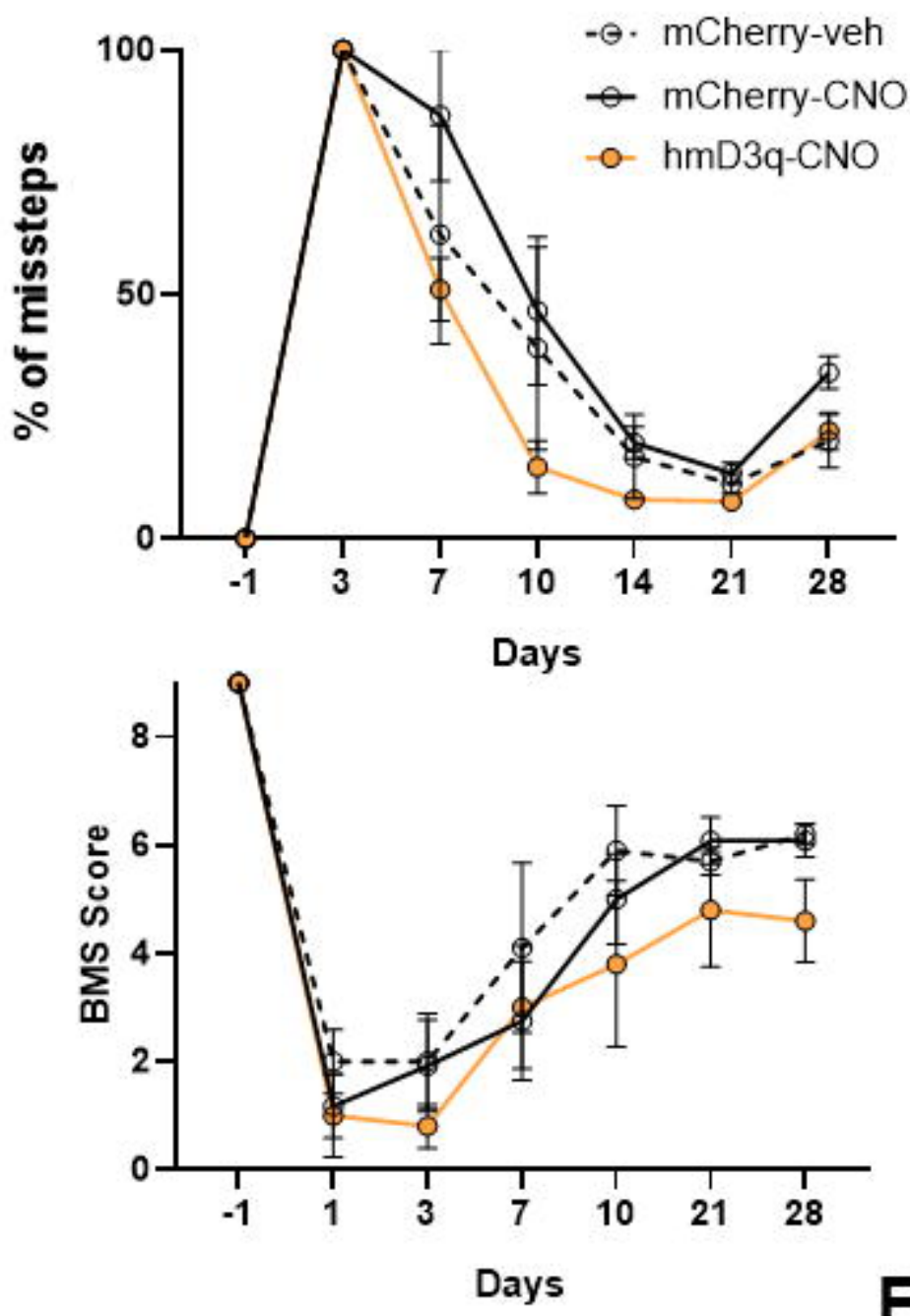

Figure 3 
A

B
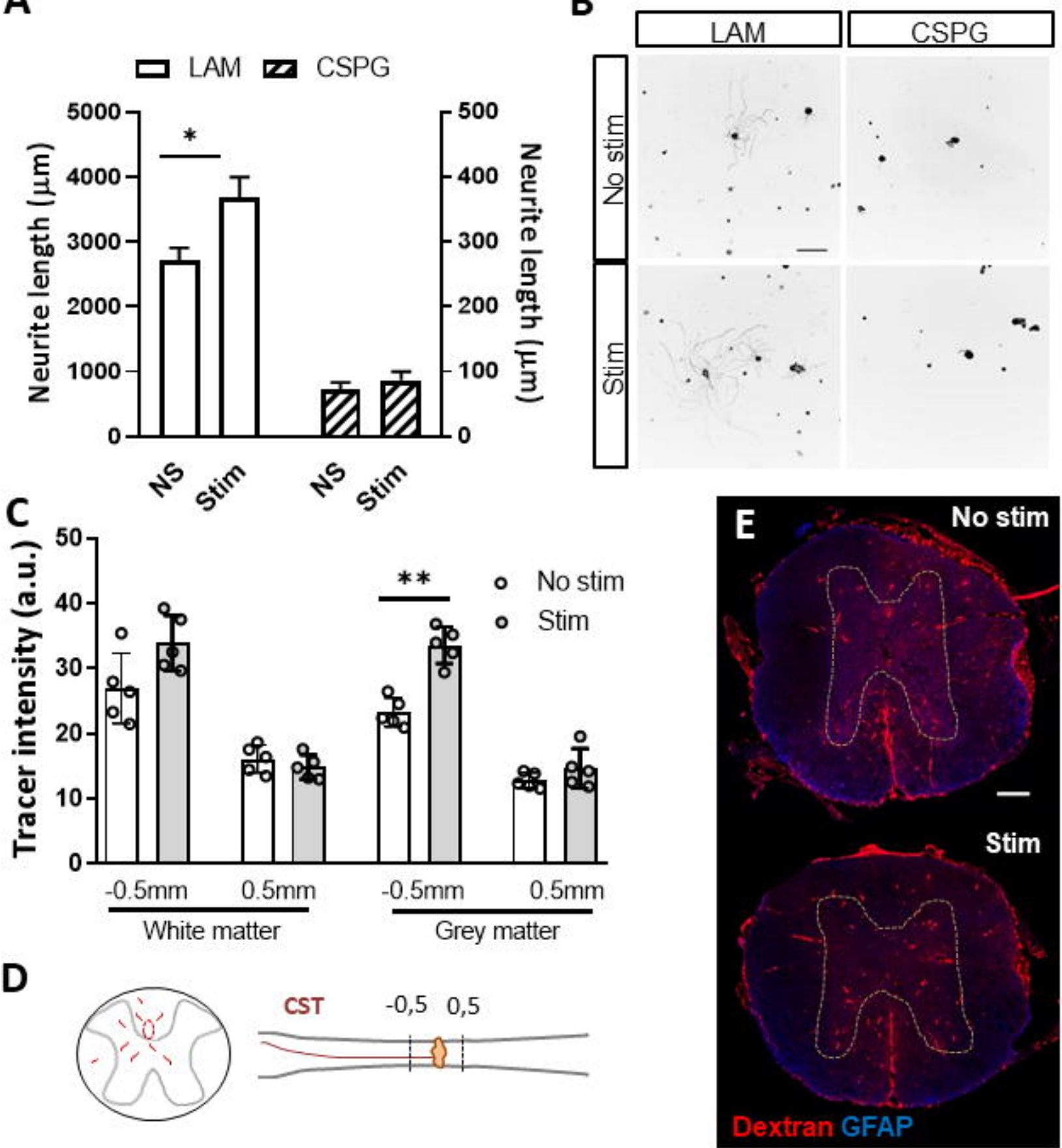

Figure 4 